\title{
PERFIL SENSORIAL DE EXTRATO HIDROSSOLÚVEL DE SOJA (Glicine Max L. Merril) COMERCIAL ADOÇADO COM SACAROSE E COM SUCRALOSE
}

\author{
EDUARTO HIDEKI TASHIMA * \\ HELENA MARIA ANDRÉ BOLINI CARDELLO **
}

\begin{abstract}
Foram comparados o perfil sensorial e curvas tempointensidade de doçura de extratos hidrossolúveis de soja comerciais, adoçados com sacarose (versão tradicional) e com edulcorante sucralose (versão "light"). A Análise Descritiva Quantitativa evidenciou que a percepção dos atributos cor creme, aroma de baunilha, aroma de soja, sabor de baunilha, sabor de soja, doçura e viscosidade foi significativamente superior para a amostra com sacarose $(p \leq 0,05)$. Não foi verificada diferença significativa entre as amostras quanto ao atributo homogeneidade. Os parâmetros da curva tempointensidade Imax, Área e Ttot para a amostra adoçada com sacarose foram de forma significativa superiores. O tempo para atingir a intensidade máxima (TImax) de doçura da sucralose no "leite" de soja é maior, ou seja, demora mais para ser percebida.
\end{abstract}

PALAVRAS-CHAVE: SOJA-ANÁLISE SENSORIAL; EDULCORANTE; EXTRATO HIDROSSOLÚVEL DE SOJA.

\section{INTRODUÇÃO}

A soja (Glicine Max L. Merril), leguminosa que emergiu como planta domesticada no Nordeste da China por volta do século XI A.C., propagou-se para grande parte do mundo (MORAIS e SILVA, 1996).

Durante séculos, o cultivo da soja permaneceu restrito apenas aos países orientais, sendo usada na preparação de grande variedade de alimentos frescos, fermentados e secos (MORAIS e SILVA, 1996).

* Bolsista Fapesp de Iniciação Científica, Faculdade de Engenharia de Alimentos (FEA), Departamento de Alimentos e Nutrição, Universidade Estadual de Campinas (UNICAMP), Campinas, SP, Brasil.

** Professora Doutora, FEA, Departamento de Alimentos e Nutrição, UNICAMP, Campinas Campinas, SP, Brasil (e-mail hellini@fea.unicamp.br). 
A soja chegou ao Ocidente no final do século XV e início do XVI. Porém, nos quatro séculos seguintes permaneceu sem muita utilização. Somente a partir de 1900 a cultura expandiu-se e ocupou posição de destaque, considerando-se recente sua introdução na cultura Ocidental.

Nas últimas décadas, houve forte propagação do cultivo de soja no Brasil que se tornou o segundo produtor mundial dessa leguminosa, sendo superado apenas pelos Estados Unidos da América (DUTRA DE OLIVEIRA et al., 1996).

A soja é considerada alimento de alto valor nutritivo e de grande importância na alimentação humana, constituindo excelente fonte de energia e proteína, além de razoável fonte de vitaminas e minerais (DUTRA de OLIVEIRA, 1981). É rica em magnésio, fósforo, ferro, cobre e zinco (MORAIS e SILVA, 1996). Quando ainda verde, apresenta alto conteúdo de niacina e de ácido ascórbico. Quando madura, torna-se ótima fonte das vitaminas $\mathrm{E}$ e $\mathrm{K}$ e boa fonte de tiamina, riboflavina $\mathrm{e}$ ácido fólico (MORAIS e SILVA, 1996).

A proteína da soja apresenta bom perfil de aminoácidos, sendo considerados limitantes apenas os sulfurados (FRANCO, 1996). Apesar da sua composição quase completa, a soja apresenta fatores que limitam sua utilização. Proteínas inibidoras da tripsina aumentam a necessidade de minerais (em parte devido à ação quelante do fitato) e vitaminas. Além disso, contém estaquinose e rafinose que são oligossacarídios não-metabolizados pelo homem.

No Ocidente, o baixo consumo de soja está relacionado ao sabor, odor e hábitos alimentares. Os sabores descritos como amargo, adstringente e rançoso, resultantes principalmente da ação da enzima lipoxigenase limitam o consumo dessa leguminosa. Nos grãos de soja íntegros, o substrato não está exposto à ação da enzima. A reação só ocorre quando os grãos se quebram e absorvem água. A ação da lipoxigenase pode ser evitada com tratamento térmico de aproximadamente $100^{\circ} \mathrm{C}$ por 5 a 10 minutos (MORAIS e SILVA, 1996).

O extrato hidrossolúvel de soja ("leite" de soja), um dos produtos da soja mais conhecidos, ainda é pouco consumido no Brasil. Os principais fatores limitantes ao consumo envolvem o sabor 
característico e a adstringência, já que em termos de aparência e valor nutritivo compara-se ao leite de vaca (MORAIS e SILVA, 1996).

Um quilo de soja fornece cerca de seis a nove litros de "leite" de soja, restando aproximadamente $700 \mathrm{~g}$ de resíduo com alto teor protéico. Sua composição química varia em função da matéria-prima utilizada e do processamento empregado. O "leite" de soja constitui boa fonte de vitamina $B$, mas contém somente $29,3 \%$ de cálcio em relação ao leite de vaca. Apresenta proteína de alto valor nutricional, sendo deficiente apenas em relação aos aminoácidos sulfurados (metionina e cistina) (DUTRA de OLIVEIRA et al., 1981).

Como o "leite" de soja não contém lactose e colesterol é ideal para portadores de intolerância à lactose ou indivíduos com indicação de ingesta de colesterol reduzida. Constitui boa fonte protéica, podendo ser utilizado na prevenção e correção da desnutrição infantil (DUTRA DE OLIVEIRA, et al., 1996). O produto industrializado pode ser encontrado no Brasil na forma original (sem aromatização), aromatizado com diferentes sabores e, ainda, adoçado com sacarose ou edulcorantes como a sucralose.

A sucralose, quimicamente denominada 1,6-dicloro-didesoxi- $\beta$ - $D$ frutofuranosil-4-cloro-4-deoxi- $\alpha$-D-galactopiranosídeo, é obtida pela cloração da sacarose de forma seletiva nas posições 4,1' e 6' da molécula do açúcar. É seiscentas vezes mais doce que a sacarose em solução a 5\% e isenta de calorias (CÂNDIDO e CAMPOS, 1996). A sucralose teve seu uso aprovado no Brasil pela Portaria $n^{\circ} 318$ do Ministério da Saúde (BRASIL, 1995), sendo produzida pela empresa JOHNSON \& JONHSON com o nome comercial Splenda. A sucralose é considerada edulcorante de alta qualidade e elevada potência, com extraordinária estabilidade e boas características físico-químicas (solubilidade em água e etanol), o que permite sua aplicação em vários alimentos e bebidas. Além disso, após inúmeros estudos clínicos, foi considerada segura para consumo humano (MILLER, 1991).

Vários adoçantes são permitidos para uso em alimentos e bebidas, os quais apresentam características específicas de intensidade, persistência do gosto doce e presença ou não de gosto residual. Essas características são determinantes na aceitação, preferência e escolha do produto por parte dos consumidores. 
O fenômeno da percepção do aroma, do gosto e da textura de alimentos, bem como das sensações térmicas e picantes não é estático. Mudanças dinâmicas em intensidade são percebidas durante todo o tempo de contato de alimentos e bebidas com a mucosa oral (BIRCH et al., 1980). A percepção da intensidade máxima de determinado composto ou ingrediente depende do tempo e da concentração do estímulo (CLIFF e HEYMANN, 1993).

A avaliação sensorial pela metodologia tempo-intensidade constitui prolongamento da análise sensorial clássica, em que são usadas escalas providas de informações temporais sobre a sensação percebida. Várias aplicações da técnica tempo-intensidade têm sido descritas na literatura, destacando-se a avaliação da doçura, entre outros atributos.

As curvas tempo-intensidade fornecem grande quantidade de informações, facilitando a comparação entre amostras de alimentos e bebidas. A técnica tempo-intensidade destacou-se por sua precisão aliada à praticidade, e tem se mostrado muito eficiente na avaliação de propriedades temporais de edulcorantes (CLIFF e HEYMANN, 1993).

O objetivo do presente estudo foi comparar o perfil sensorial e curvas tempo-intensidade de doçura de dois extratos hidrossolúveis comerciais de soja, sendo um adoçado com sacarose e o outro com edulcorante (sucralose).

\section{MATERIAL E MÉTODOS}

\subsection{MATERIAL}

Foram utilizadas amostras de extrato hidrossolúvel de soja ("leite" de soja) nas versões tradicional (com sacarose) e "light" (com sucralose).

\subsection{DETERMINAÇÕES FÍSICO-QUÍMICAS}

As determinações de $\mathrm{pH}$, sólidos solúveis, sólidos totais, acidez total titulável, açúcares redutores e não-redutores foram realizadas de acordo com a AOAC (1995). 
Utilizou-se colorímetro Hunter para determinação da coloração das amostras de extrato hidrossolúvel de soja, obtendo-se as leituras de "L", "a" e "b" (AOAC, 1995).

\subsection{ANÁLISE SENSORIAL}

\subsubsection{Análise Descritiva Quantitativa}

Dezesseis candidatos, pré-selecionados pela habilidade em discriminar diferenças entre amostras de extrato hidrossolúvel de soja (análise seqüencial), efetuaram o levantamento dos termos descritivos (MEILGAARD e CIVILLE, 1999).

Os provadores (pré-selecionados) iniciaram a Análise Descritiva Quantitativa com o desenvolvimento dos termos descritivos das amostras pelo método de rede de Kelly (MOSKOWITZ, 1983), recebendo amostras aos pares e listando em ficha apropriada as similaridades e diferenças entre as amostras apresentadas.

A equipe reuniu-se quatro vezes e escolheu, em debate aberto, os termos mais apropriados para descrever os atributos das amostras. Foram, então, elaboradas fichas de avaliação, com escalas nãoestruturadas de 9 centímetros, ancoradas nos pontos extremos com termos de intensidade.

Várias sessões de treinamento foram realizadas para que as notas dos provadores ficassem na mesma região da escala, mediante apresentação de amostras-referência dos extremos das escalas, sendo as referências escolhidas em função dos termos descritivos e do consenso da equipe.

\subsubsection{Seleção dos Provadores}

Para a seleção da equipe definitiva utilizou-se a ficha elaborada com as escalas de intensidade para os termos definidos. Os provadores foram selecionados com base nas características de poder de discriminação entre as amostras, repetibilidade e concordância com a equipe (DAMÁSIO e COSTEL, 1991). 
Efetuou-se análise de variância (ANOVA) com duas fontes de variação (amostra e repetição) para cada atributo e cada provador. Foram selecionados os provadores que obtiveram valores de $F_{\text {amostra }}$ significativo

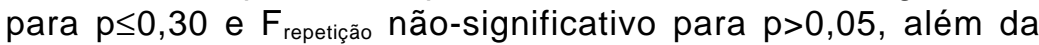
concordância das médias com as da equipe (valores de médias de cada provador na mesma ordem e próximos à média da equipe para cada atributo avaliado).

\subsubsection{Avaliação das Amostras}

Os provadores selecionados e treinados participaram dos testes, sendo as amostras servidas em cabines individuais, usando-se copos plásticos descartáveis com capacidade para $20 \mathrm{~mL}$, codificados com algarismos de três dígitos.

Todas as amostras foram apresentadas de forma monádica, com quatro repetições aleatorizadas, num total de 8 sessões.

\subsubsection{Análise dos Dados}

Avaliaram-se os dados pela ANOVA de dois fatores (amostra e provadores) com interação para cada atributo, sendo aplicado o teste de Tukey para comparação das médias das amostras, bem como realizada a Análise de Componentes Principais.

As análises estatísticas foram efetuadas mediante programas do pacote estatístico SAS (2001).

\subsubsection{Análise Tempo-Intensidade}

As análises tempo-intensidade das amostras de extrato hidrossolúvel de soja, nas versões tradicional e "light" foram realizadas com o programa Sistema de Coleta de Dados Tempo-Intensidade (SCDTI), desenvolvido no Laboratório de Análise Sensorial da Faculdade de Engenharia de Alimentos da Universidade Estadual de Campinas (CARDELLO et al., 1996). 


\subsubsection{Seleção dos Provadores}

Candidatos pré-selecionados pela análise seqüencial, com testes triangulares de diferença e habilidade em trabalhar com computador, foram escolhidos para participar dos testes.

Na segunda etapa da pré-seleção, os candidatos capacitados a operar o programa (interagindo a sensação percebida com o registro da mesma por meio do "mouse") foram escolhidos pelo poder de discriminação entre as amostras, repetibilidade e concordância com a equipe para 0 gosto doce existente nas amostras.

Efetuou-se intenso treinamento com os provadores para familiarizaremse com o programa e padronização da metodologia.

Cada provador pré-selecionado realizou o teste tempo-intensidade das amostras em relação à doçura, as quais foram apresentadas de forma monádica com 4 repetições. Os dados foram analisados pela ANOVA com as seguintes fontes de variação: amostra e repetição para cada parâmetro das curvas, para cada provador e para o estímulo doce. Foram então obtidos os valores para $F_{\text {amostra }}$ e $F_{\text {repetição }}$ para cada provador. Os provadores com $F_{\text {amostra }}$ significativo para $p \leq 0,30$ e $F_{\text {repetição }}$ não-significativo para p>0,05 e concordância das médias com as da equipe (valores de médias de cada provador próximo à média da equipe) foram selecionados.

\subsubsection{Avaliação das Amostras}

Dos dez candidatos pré-selecionados, seis foram selecionados e treinados para a execução dos testes. As amostras foram apresentadas de forma monádica (STONE e SIDEL, 1993) e aleatorizada, com 4 repetições, em sala climatizada $\left(22^{\circ} \mathrm{C}\right)$.

Em todos os testes, os provadores receberam $5,0 \mathrm{~mL}$ da amostra a ser avaliada em copos plásticos descartáveis com capacidade para $50 \mathrm{~mL}$, codificados com algarismos de três dígitos. O provador devia colocar todo volume da amostra na boca e seguir as instruções dadas pelo programa. Assim, ao sinal emitido pelo computador, juntamente com o sinal gráfico, o provador devia colocar a amostra na boca e 
ingeri-la no tempo previamente determinado. Durante tal procedimento, o provador deveria registrar com o "mouse" toda intensidade de estímulo percebido em escala na tela do monitor.

Os tempos de espera inicial e de permanência da amostra na boca (10 segundos), o tempo após a ingestão e a duração total dos testes (45 segundos) foram padronizados e seguidos rigorosamente. O tempo total de duração dos testes variou em função da concentração de equivalência.

A escala utilizada (uma vez que o programa possibilita a escolha) foi de 9 pontos, na qual 0 corresponde a nenhum, 4,5 a moderado e 9 a forte.

\subsubsection{Parâmetros da Curva Tempo-Intensidade}

A partir de cada curva tempo-intensidade obtida, os parâmetros coletados incluíram Tempo para atingir a intensidade máxima (TImax), Área sob a curva (Área), Tempo total de duração do estímulo (Ttot) e Intensidade máxima do estímulo (Imax) (BONNANS e NOBLE, 1993; KETELSEN et al., 1993).

\subsubsection{Análise dos Dados}

Os valores dos parâmetros das curvas tempo-intensidade coletados foram submetidos à ANOVA de dois fatores (amostra e provadores) $\mathrm{e}$ interação para cada parâmetro. Aplicou-se o teste de Tukey para comparação das médias, efetuando-se também a Análise de Componentes Principais.

As análises estatísticas foram realizadas usando-se os programas do pacote estatístico SAS (2001).

\section{RESULTADOS E DISCUSSÃO}

\subsection{DETERMINAÇÕES FÍSICO-QUÍMICAS}

Os valores das médias e desvios-padrão encontrados para pH, sólidos 
solúveis, sólidos totais, acidez total titulável e açúcares redutores e não-redutores estão expressos na Tabela 1. É possível verificar que as amostras não diferiram em relação ao $\mathrm{pH}$ e à acidez total titulável (considerando-se o desvio-padrão). A amostra com sacarose apresentou maior quantidade de sólidos solúveis e de sólidos totais.

\section{TABELA 1 - MÉDIAS DOS VALORES DE PH, SÓLIDOS SOLÚVEIS, SÓLIDOS TOTAIS E ACIDEZ TOTAL TITULÁVEL DAS AMOSTRAS DE EXTRATO HIDROSSOLÚVEL DE SOJA ADOÇADO COM SACAROSE (TRADICIONAL) E COM SUCRALOSE (“LIGHT”)}

\begin{tabular}{|c|c|c|}
\hline \multirow{2}{*}{$\begin{array}{l}\text { Determina I es } \\
\text { F sico-Qu micas }\end{array}$} & \multicolumn{2}{|c|}{ AMOSTRA } \\
\hline & Tradicional & Light \\
\hline $\mathrm{pH}$ & 7,0 & 7,0 \\
\hline S lidos Soløreis* ( Brix) & $10,0 \pm 0,5$ & $7,2 \pm 0,3$ \\
\hline$S$ lidos Totais* $(\mathrm{g} / 100 \mathrm{~mL})$ & $11,151 \pm 0,077 \mathrm{~g} / 100 \mathrm{~mL}$ & $(6,825 \pm 0,387) \mathrm{g} / 100 \mathrm{~mL}$ \\
\hline Acidez Total Titul/Æ/el* & $0,063 \pm 0,004$ & $0,059 \pm 0,007$ \\
\hline ( $\mathrm{mL}$ de /Eido $\mathrm{c}$ trico anidro/100 $\mathrm{mL}$ ) & & \\
\hline A œares redutores (\% p/v) & $3,7 \pm 0,4$ & $1,9 \pm 0,3$ \\
\hline A oeares $n^{a}$ o-redutores (\% $\left.\mathrm{p} / \mathrm{v}\right)$ & $6,8 \pm 0,2$ & $6,1 \pm 0,2$ \\
\hline
\end{tabular}

*Média \pm desvio-padrão.

As médias e desvios-padrão dos valores encontrados na determinação de cor estão expressos na Tabela 2. Analisando-se os resultados podese perceber que não houve diferença significativa em relação à cor para as amostras adoçadas com sacarose e com sucralose e suas repetições.

\section{TABELA 2-MÉDIAS E DESVIOS-PADRÃO DOS VALORES ENCONTRADOS NA DETERMINAÇÃO DE COR}

\begin{tabular}{l|c|c|c}
\hline \multirow{2}{*}{ Tradicional } & $\mathrm{L}$ & $\mathrm{A}$ & $\mathrm{b}$ \\
\cline { 2 - 4 } "Light" & $73,22^{\mathrm{a}} \pm 0,30$ & $0,80^{\mathrm{b}} \pm 0,06$ & $12,13^{\mathrm{a}} \pm 0,03$ \\
\hline
\end{tabular}

$\mathrm{L}=$ Luminosidade.

$\mathrm{a}=$ Parâmetro de cor que vai do verde ao vermelho.

$\mathrm{b}=$ parâmetro de cor que vai do amarelo ao azul. 


\subsubsection{Análise Descritiva Quantitativa}

Dos 21 candidatos, 16 foram pré-selecionados e participaram das sessões do levantamento dos termos descritores. Os atributos escolhidos em consenso pelos membros da equipe sensorial para a avaliação das amostras de extrato hidrossolúvel de soja foram: coloração creme, aroma de baunilha, aroma de soja, sabor de baunilha, sabor de soja, sabor de baunilha, doçura, viscosidade e homogeneidade. As definições e as referências para cada termo descritor encontramse no Quadro 1.

\section{QUADRO 1 - DEFINIÇÕES E REFERÊNCIAS PARA OS TERMOS DESCRITORES LEVANTADOS PELOS PROVADORES}

\begin{tabular}{|c|c|c|}
\hline $\begin{array}{l}\text { Termo Descritor } \\
\text { (Atributo) }\end{array}$ & Defini ${ }^{a} 0$ & Referव̄cias \\
\hline $\begin{array}{l}\text { Do ura } \\
\text { (DOC) }\end{array}$ & $\begin{array}{l}\text { Gosto doce caracter stico da solu }{ }^{\stackrel{a}{0}} 0 \\
\text { de sacarose }\end{array}$ & $\begin{array}{l}\text { Pouca: } 50 \mathrm{~mL} \text { de extrato hidrossolorel de } \\
\text { soja tradicional adicionado de } 75 \mathrm{~mL} \text { de leite } \\
\text { desnatado marca Parmalat } \\
\text { Muita: } 100 \mathrm{~mL} \text { de extrato hidrossolorel de } \\
\text { soja tradicional com } 4 \mathrm{~g} \text { de sacarose. }\end{array}$ \\
\hline $\begin{array}{l}\text { Sabor de soja } \\
\text { (SSOJ) }\end{array}$ & $\begin{array}{l}\text { Sabor caracter stico do extrato de } \\
\text { soja marca Mais Vita }\end{array}$ & $\begin{array}{l}\text { Fraco: } 50 \mathrm{~mL} \text { de extrato hidrossolo el de } \\
\text { soja tradicional com } 50 \mathrm{~mL} \text { de leite desnatado } \\
\text { Parmalat } \\
\text { Forte: } 75 \mathrm{~mL} \text { de extrato hidrossolorel de } \\
\text { soja tradicional com } 4 \mathrm{~g} \text { de extrato de soja } \\
\text { Mais Vita }\end{array}$ \\
\hline $\begin{array}{l}\text { Aroma de soja } \\
\text { (ASOJ) }\end{array}$ & $\begin{array}{l}\text { Aroma caracter stico do extrato de } \\
\text { soja marca Mais Vita }\end{array}$ & $\begin{array}{l}\text { Fraco: } 50 \mathrm{~mL} \text { de extrato hidrossolo el de } \\
\text { soja tradicional com } 50 \mathrm{~mL} \text { de leite desnatado } \\
\text { marca Parmalat } \\
\text { Forte: } 50 \mathrm{~mL} \text { de extrato hidrossolo el de } \\
\text { soja tradicional com } 5 \mathrm{~g} \text { de extrato de soja } \\
\text { marca Mais Vita }\end{array}$ \\
\hline $\begin{array}{l}\text { Sabor de } \\
\text { baunilha } \\
\text { (SBAU) }\end{array}$ & $\begin{array}{l}\text { Sabor caracter stico do aroma } \\
\text { artificial de baunilha marca Oetker }\end{array}$ & $\begin{array}{l}\text { Fraco : } 1 \mathrm{~mL} \text { de aroma artificial de baunilha } \\
\text { Oetker em } 400 \mathrm{~mL} \text { de Agua filtrada } \\
\text { Forte : } 1 \mathrm{~mL} \text { de aroma artificial de baunilha } \\
\text { Oetker em } 100 \mathrm{~mL} \text { de Agua filtrada }\end{array}$ \\
\hline $\begin{array}{l}\text { Aroma de } \\
\text { baunilha } \\
\text { (ABAU) }\end{array}$ & $\begin{array}{l}\text { Aroma caracter stico do aroma } \\
\text { artificial de baunilha marca Oetker }\end{array}$ & $\begin{array}{l}\text { Fraco: } 1 \mathrm{~mL} \text { aroma artificial de baunilha } \\
\text { Oetker em } 100 \mathrm{~mL} \text { Agua filtrada } \\
\text { Forte : } 1 \mathrm{~mL} \text { aroma artificial de baunilha } \\
\text { Oetker em } 25 \mathrm{~mL} \text { de Agua filtrada }\end{array}$ \\
\hline $\begin{array}{l}\text { Viscosidade } \\
\text { (VISC) }\end{array}$ & $\begin{array}{l}\text { Quanto o produto se adere } \mathrm{s} \\
\text { paredes do recipiente que o contøm }\end{array}$ & $\begin{array}{l}\text { Pouca : } 50 \mathrm{~mL} \text { de extrato hidrossolo el de } \\
\text { soja tradicional em } 50 \mathrm{~mL} \text { de Agua filtrada } \\
\text { Muita: } 50 \mathrm{~mL} \text { de extrato hidrossolo el de } \\
\text { soja tradicional com } 5 \mathrm{~g} \text { de extrato de soja } \\
\text { Mais Vita }\end{array}$ \\
\hline $\begin{array}{l}\text { Homogeneidade } \\
\text { (HOMO) }\end{array}$ & $\begin{array}{l}\text { o quanto a aparCícia se assemelha } \\
\text { ao longo de todo o produto }\end{array}$ & $\begin{array}{l}\text { Pouca : } 50 \mathrm{~mL} \text { de extrato hidrossolorel de } \\
\text { soja tradicional com } 2 \mathrm{~g} \text { de extrato de soja } \\
\text { Mais Vita } \\
\text { Muita : } 50 \mathrm{~mL} \text { de leite desnatado Parmalat }\end{array}$ \\
\hline $\begin{array}{l}\text { Cor creme } \\
\text { (COR) }\end{array}$ & $\begin{array}{l}\text { Cor caracter stica de } \\
\text { condensado Mo a Nestl } \varnothing\end{array}$ & $\begin{array}{l}\text { Fraco: } 50 \mathrm{~mL} \text { de leite desnatado Parmalat } \\
\text { Forte: } 100 \mathrm{~mL} \text { de extrato hidrossoløel de } \\
\text { soja tradicional com } 0,15 \mathrm{~g} \text { de caf } \varnothing \text { soløel } \\
\text { granulado Nescaf } \varnothing\end{array}$ \\
\hline
\end{tabular}


De acordo com os critérios estabelecidos foram selecionados 10 provadores, dos 16 candidatos pré-selecionados. Nas Tabelas 3 e 4 são apresentados os valores de $p$ (níveis de significância) para $F_{\text {amostra }}$ e $F_{\text {repetição }}$ respectivamente, para cada um dos provadores. Notou-se que os provadores que revelaram menor poder de discriminação também apresentaram baixa repetibilidade.

\section{TABELA 3 - NÍVEIS DE SIGNIFICÂNCIA (p) PARA PROVADORES EM FUNÇÃO DE DISCRIMINAÇÃO DAS AMOSTRAS ( $\left.F_{\text {amostra }}\right)$}

\begin{tabular}{lllllllll}
\hline Provador & HOMO & VISC & COR & ASOJ & ABAU & DOC & SBAU & SSOJ \\
\hline P1 & 0,0673 & 0,0033 & 0,1325 & 0,0065 & 0,0125 & 0,1598 & 0,1254 & 0,0987 \\
P2 & 0,2193 & 0,1141 & 0,1225 & 0,0158 & 0,0023 & 0,2654 & 0,0258 & 0,0758 \\
P3 & 0,0064 & 0,0082 & 0,2545 & 0,2584 & 0,0025 & 0,2356 & 0,0059 & 0,0522 \\
P4 & 0,0067 & 0,0125 & 0,2456 & 0,0158 & 0,0158 & 0,2563 & 0,0068 & 0,2583 \\
P5 & 0,1025 & 0,0012 & 0,2356 & 0,0031 & 0,2987 & 0,1523 & 0,0265 & 0,2835 \\
P6 & 0,0501 & 0,0123 & 0,2589 & 0,0526 & 0,2584 & 0,0156 & 0,0954 & 0,0089 \\
P7 & 0,0060 & 0,1217 & 0,1478 & 0,0036 & 0,2635 & 0,0032 & 0,0852 & 0,0098 \\
P8 & 0,0300 & 0,1181 & 0,1598 & 0,0015 & 0,2588 & 0,0065 & 0,0842 & 0,0584 \\
P9 & 0,0523 & 0,1585 & 0,1254 & 0,1265 & 0,0265 & 0,0096 & 0,0056 & 0,0951 \\
P10 & 0,2197 & 0,1695 & 0,2369 & 0,2543 & 0,0012 & 0,1582 & 0,0295 & 0,0258 \\
P11* & 0,4590 & 0,4388 & 0,8478 & 0,9911 & 0,3421 & 0,9536 & 0,6362 & 0,4804 \\
P12* & 0,4828 & 0,5486 & 0,6852 & 0,4752 & 0,3584 & 0,4125 & 0,8526 & 0,5842 \\
P13* & 0,4429 & 0,5246 & 0,3489 & 0,6985 & 0,7851 & 0,7421 & 0,7854 & 0,3547 \\
P14* & 0,5862 & 0,4258 & 0,4587 & 0,4756 & 0,5284 & 0,8560 & 0,3541 & 0,3698 \\
P15* $^{*}$ & 0,3589 & 0,3658 & 0,8965 & 0,4856 & 0,8695 & 0,5841 & 0,4562 & 0,4521 \\
P16 $^{*}$ & 0,8596 & 0,8586 & 0,6358 & 0,5986 & 0,4859 & 0,5268 & 0,4257 & 0,3145 \\
\hline
\end{tabular}

* Provadores com valores de $p$ de Famostra $>0,30$ que não foram selecionados. $\mathrm{HOMO}=$ Homogeneidade VISC = Viscosidade COR = Cor Creme; $\mathrm{ASO}=\mathrm{J}=$ Aroma de Soja; $\mathrm{ABAU}=$ Aroma de Baunilha; $\mathrm{DOC}=$ Doçura; SBAU = Sabor de Baunilha; SSOJ = Sabor de Soja.

3.2.1.2 Análise Descritiva Quantitativa das Amostras de Extrato Hidrossolúvel de Soja adoçado com Sacarose (Versão tradicional) e com Sucralose (Versão "Light")

A análise de variância dos resultados (fontes de variação: provador, amostra e interação entre amostra e provador) mostrou que houve diferença significativa $(p \leq 0,0001)$ entre as amostras em relação a todos os atributos avaliados. 
A partir da diferença mínima significativa (DMS) obtida pelo teste de médias de Tukey $(p \leq 0,05)$ foi realizada a comparação entre as médias (Tabela 5).

\section{TABELA 4 - NÍVEIS DE SIGNIFICÂNCIA (p) PARA PROVADORES EM FUNÇÃO DA REPETIBILIDADE ( $\left.F_{\text {repetição }}\right)$}

\begin{tabular}{crrrrrrrr}
\hline Provador & HOMO & VISC & COR & ASOJ & ABAU & DOC & SBAU** & SSOJ \\
\hline P 1 & 0,0893 & 0,8375 & 0,9536 & 0,0501 & 0,5645 & 0,4454 & 0,7945 & 0,0689 \\
P 2 & 0,5585 & 0,6130 & 0,5903 & 0,0652 & 0,5624 & 0,5487 & 0,2155 & 0,5452 \\
P 3 & 0,1025 & 0,2197 & 0,5876 & 0,0548 & 0,0974 & 0,4512 & 0,1544 & 0,9856 \\
P 4 & 0,4388 & 0,5519 & 0,5273 & 0,4751 & 0,5697 & 0,1531 & 0,0547 & 0,0689 \\
P 5 & 0,5273 & 0,4564 & 0,0673 & 0,1258 & 0,3360 & 0,0712 & 0,5115 & 0,4846 \\
P 6 & 0,4347 & 0,1250 & 0,2577 & 0,0654 & 0,5468 & 0,6512 & 0,3374 & 0,4568 \\
P 7 & 0,6130 & 0,0634 & 0,1091 & 0,7895 & 0,2145 & 0,0548 & 0,6362 & 0,0698 \\
P 8 & 0,5000 & 0,4773 & 0,3152 & 0,8519 & 0,6651 & 0,1250 & 0,6548 & 0,4646 \\
P 9 & 0,5000 & 0,5735 & 0,0631 & 0,4683 & 0,8445 & 0,4034 & 0,0801 & 0,5456 \\
P 10 & 0,4561 & 0,6123 & 0,8918 & 0,1523 & 0,0506 & 0,7848 & 0,9536 & 0,1548 \\
P11* & 0,0437 & 0,0458 & 0,0258 & 0,0158 & 0,0452 & 0,0088 & 0,0268 & 0,0227 \\
P12* & 0,0258 & 0,0257 & 0,0065 & 0,0169 & 0,0255 & 0,0456 & 0,0154 & 0,0264 \\
P13* & 0,0465 & 0,0355 & 0,0226 & 0,0269 & 0,0034 & 0,0098 & 0,0095 & 0,0075 \\
P14 & 0,0368 & 0,0166 & 0,0472 & 0,0354 & 0,0014 & 0,0425 & 0,0062 & 0,0456 \\
P15* & 0,0358 & 0,0353 & 0,0061 & 0,0418 & 0,0168 & 0,0364 & 0,0346 & 0,0499 \\
P16* & 0,0327 & 0,0362 & 0,0488 & 0,0155 & 0,0484 & 0,0042 & 0,0333 & 0,0368
\end{tabular}

* Provadores com valores de $p$ de Famostra $>0,30$ que não foram selecionados.

HOMO = Homogeneidade; VISC = Viscosidade; COR = Cor Creme; ASOJ = Aroma de Soja; $\mathrm{ABAU}=$ Aroma de Baunilha; $\mathrm{DOC}=$ Doçura; SBAU = Sabor de Baunilha; SSOJ = Sabor de Soja.

\section{TABELA 5 - MÉDIAS DOS ATRIBUTOS PARA AS AMOSTRAS DE “LEITE” DE SOJA}

\begin{tabular}{c|c|c|c|c|c|c|c|c}
\hline Amostra & HOMO & VISC & COR & ASOJ & ABAU & DOC & SBAU & SSOJ \\
\hline Tradicional & $6,6900^{\mathrm{a}}$ & $4,8633^{\mathrm{a}}$ & $4,9567^{\mathrm{a}}$ & $4,9033^{\mathrm{a}}$ & $6,3167^{\mathrm{a}}$ & $4,2333^{\mathrm{a}}$ & $4,3167^{\mathrm{a}}$ & $5,6000^{\mathrm{a}}$ \\
\hline Light & $6,6233^{\mathrm{a}}$ & $3,5533^{\mathrm{b}}$ & $3,4600^{\mathrm{b}}$ & $4,2733^{\mathrm{b}}$ & $3,9567^{\mathrm{b}}$ & $3,4467^{\mathrm{b}}$ & $2,9567^{\mathrm{b}}$ & $4,6200^{\mathrm{b}}$ \\
\hline DMS & 0,3594 & 0,2657 & 0,259 & 0,4256 & 0,3106 & 0,2218 & 0,2402 & 0,327 \\
\hline
\end{tabular}

* Médias seguidas por letras iguais na mesma coluna não diferem entre si.

$\mathrm{HOMO}=$ Homogeneidade; VISC = Viscosidade; $\mathrm{COR}=$ Cor Creme; ASOJ = Aroma de Soja; $\mathrm{ABAU}=$ Aroma de Baunilha; $\mathrm{DOC}=$ Doçura; SBAU = Sabor de Baunilha; SSOJ = Sabor de Soja.

A percepção dos atributos viscosidade, cor creme, aroma de soja, 
aroma de baunilha, doçura, sabor de baunilha e sabor de soja foi significativamente superior $(p \leq 0,05)$ para a amostra adoçada com sacarose. Tal fato indica que, provavelmente, a substituição da sacarose pela sucralose promove diminuição na percepção desses atributos, com exceção da homogeneidade.

Verificou-se correlação inversa entre a cor avaliada sensorialmente e o valor medido instrumentalmente (Tabela 2 ).

Os resultados da análise de componentes principais (ACP) está representado na Figura 1.

FIGURA1 - FIGURA BIDIMENSIONAL DA ANÁLISE DOS COMPONENTES PRINCIPAIS DAS AMOSTRAS DE “LEITE” DE SOJA

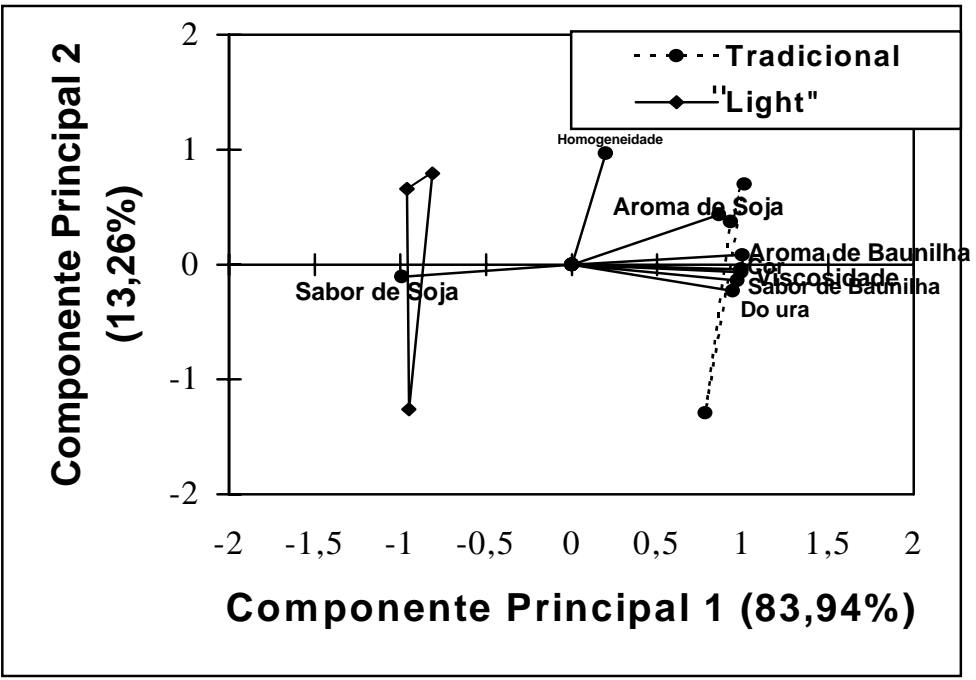

Verificou-se que $83,94 \%$ da variação ocorrida entre as amostras foi explicada pelo primeiro eixo (Componente Principal 1). Os Componentes Principais 1 e 2 explicaram juntos, 97,20\% da variação entre as amostras. 
De acordo com MUÑOZ et al. (1986), os vetores com medidas mais distantes de zero correspondem às variáveis com maior influência sobre o valor do componente principal. Vetores mais próximos de zero indicam variável com pequena influência sobre o componente principal. Os atributos viscosidade, cor, doçura, aroma de soja, aroma e sabor de baunilha (positivamente) e sabor de soja (negativamente) contribuíram com maior peso sobre a variabilidade associada a esse eixo. $\mathrm{O}$ atributo homogeneidade exerceu maior influência sobre o Componente Principal 2 (positivamente).

As amostras distinguiram-se pelas localizações bem-definidas no gráfico bidimensional. $\mathrm{O}$ experimento apresentou ótima repetibilidade, evidenciada pelo agrupamento dos pontos representativos de cada amostra.

A amostra de "leite" de soja adoçada com sacarose caracterizou-se principalmente pelos atributos viscosidade, cor, doçura, aroma de soja, aroma de baunilha e sabor de baunilha. Os valores das intensidades desses mesmos atributos para a amostra com sacarose mostraramse superiores à amostra com sucralose (Tabela 5).

A amostra de "leite" de soja adoçada com sucralose caracterizou-se principalmente pelo atributo sabor de soja, cujo valor mostrou-se significativamente superior ao da amostra com sacarose (Tabela 5).

Como os vetores aroma de soja, aroma de baunilha, sabor de baunilha, cor, doçura e viscosidade em relação ao sabor de soja estão em direções opostas é possível sugerir que existe correlação linear negativa entre o sabor de soja e os demais. Essas observações foram comprovadas pelos valores de coeficiente de correlação entre tais atributos, sempre superiores a 0,9 e no caso de correlação negativa sempre acima de $-0,9$.

\subsubsection{Análise Tempo-Intensidade}

As curvas tempo-intensidade registradas para o estímulo doce das amostras com sacarose e com sucralose seguiram a mesma tendência. As características destas curvas estão representadas nas Figuras 2 e 3 , tomando-se como exemplo os registros de determinado 
provador e suas três repetições. A análise de variância dos resultados (fontes de variação: provador, amostra e interação entre amostra e provador) mostrou que houve diferença significativa $(p \leq 0,0001)$ entre as duas amostras em relação a todos os parâmetros da curva avaliados. Foi realizado teste de Tukey para comparação dessas médias e o resultado encontra-se na Tabela 6.

FIGURA 2 - CURVA TEMPO-INTENSIDADE CARACTERÍSTICA DO GOSTO DOCE DA AMOSTRA DE “LEITE” DE SOJA ADOÇADA COM SACAROSE

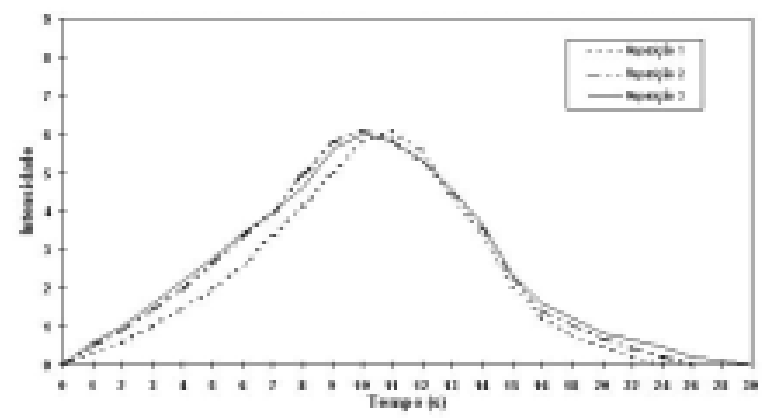

\section{FIGURA 3 - CURVA TEMPO-INTENSIDADE CARACTERÍSTICA DO GOSTO DOCE DA AMOSTRA DE “LEITE” DE SOJA ADOÇADA COM SUCRALOSE}

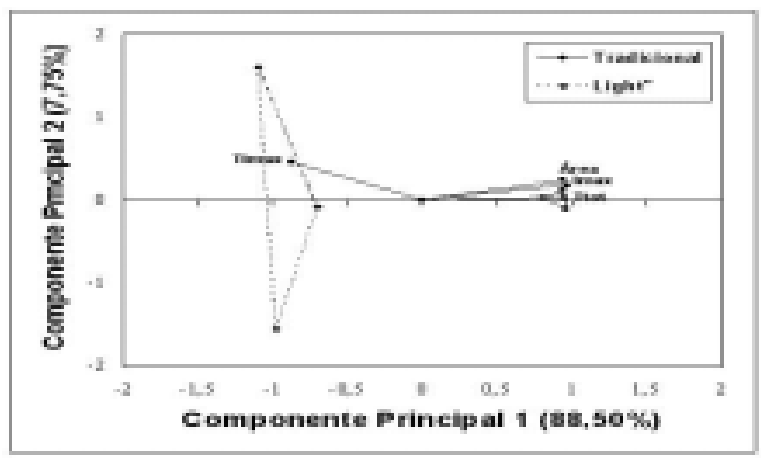




\section{TABELA 6 - MÉDIAS DOS PARÂMETROS DAS CURVAS TEMPO- INTENSIDADE PARA O GOSTO DOCE DAS AMOSTRAS DE “LEITE” DE SOJA TRADICIONAL E "LIGHT"}

\begin{tabular}{l|c|c|c|c}
\hline Amostra & Timax (s) & rea & Ttot (s) & Imax \\
\hline Tradicional & $7,0237^{\mathrm{b}}$ & $81,968^{\mathrm{a}}$ & $25,0357^{\mathrm{a}}$ & $5,42950^{\mathrm{a}}$ \\
\hline Light & $8,7410^{\mathrm{a}}$ & $65,018^{\mathrm{b}}$ & $21,7030^{\mathrm{b}}$ & $4,73847^{\mathrm{D}}$ \\
\hline DMS & 0,6851 & 8,7798 & 1,7773 & 0,1672 \\
\hline
\end{tabular}

TImax = Tempo para atingir a intensidade máxima; Ttot = Tempo total de duração do estímulo; Imax = Intensidade máxima.

A amostra adoçada com sacarose apresentou valores de Área, Ttot e Imax significativamente superiores $(p \leq 0,05)$ aos da amostra com sucralose. No entanto, o TImax da amostra "light" mostrou-se significativamente superior $(p \leq 0,05)$ ao da amostra com sacarose.

O resultado da análise de componentes principais (ACP) está representado na Figura 4. A distinção entre as amostras pode ser observada pela localização bem-definida de cada uma no gráfico.

Verificou-se que $88,50 \%$ da variação ocorrida entre as amostras foi explicada pelo primeiro eixo (Componente Principal 1) e 7,75\% pelo segundo (Componente Principal 2). Os Componentes Principais 1 e 2 explicaram juntos, $96,25 \%$ da variação entre as amostras.

A amostra com sacarose caracterizou-se pelos parâmetros Área, Ttot e Imax, enquanto a com sucralose pelo parâmetro Timax, principalmente.

A proximidade entre os vetores Imax, Ttot e Área indicou correlação positiva entre os parâmetros das amostras (coeficiente de correlação linear $-r>0,95)$. 
FIGURA 4- FIGURA BIDIMENSIONAL DA ANÁLISE DE COMPONENTES PRINCIPAIS DOS PARÂMETROS DAS CURVAS TEMPO-INTENSIDADE PARA O GOSTO DOCE DAS AMOSTRAS

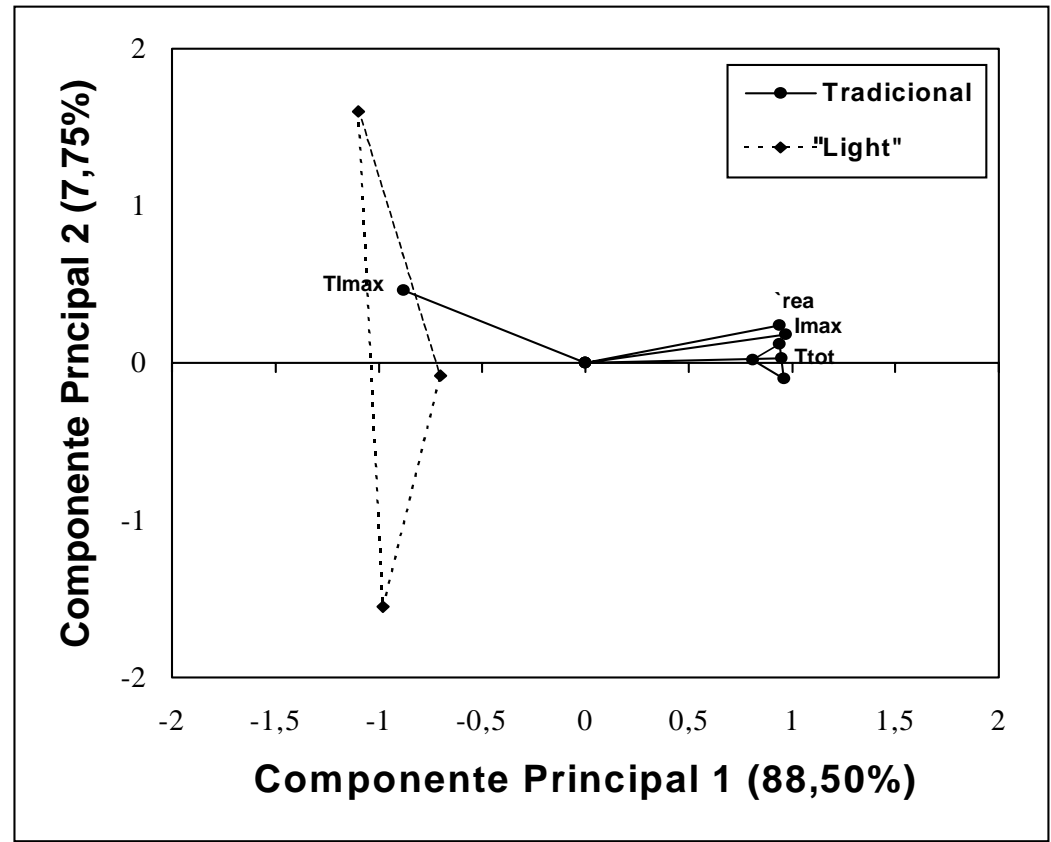

Os resultados obtidos na Análise Tempo-Intensidade e na Análise Descritiva Quantitativa foram concordantes em relação à doçura. Explicaram o resultado referente ao descritor "doçura", cujos parâmetros da curva tempo-intensidade Imax, Área e Ttot para a amostra adoçada com sacarose foram de forma significativa, superiores. O tempo para atingir a intensidade máxima (TImax) de doçura do "leite" de soja com sucralose mostrou-se maior (demorou mais para ser percebida), no entanto foi mais prolongada.

\section{CONCLUSÃO}

A Análise Descritiva Quantitativa evidenciou que a percepção dos 
atributos cor creme, aroma de baunilha, aroma de soja, sabor de baunilha, sabor de soja, doçura e viscosidade foi significativamente superior para o "leite" de soja adoçado com sacarose. Não foi verificada diferença significativa entre as amostras de "leite" de soja quanto ao atributo homogeneidade.

As curvas tempo-intensidade comprovaram os resultados obtidos na análise descritiva e indicaram que os parâmetros da Imax, Área e Ttot para a amostra adoçada com sacarose foram significativamente superiores. Também evidenciaram que o tempo para atingir a intensidade máxima (TImax) de doçura da sucralose no "leite" de soja é maior, ou seja, demora mais para ser percebida.

\section{Abstract}

\section{SENSORY PROFILE OF COMMERCIAL HIDROSSOLUBLE SOY EXTRACT} (Glicine Max L. Merril) SWEETENED WITH SUCROSE AND SUCRALOSE

The sensorial profile and time-intensity curves of sweetness of commercial hidrossoluble soy extracts, sweetened with sucrose (traditional version) and edulcorant sucralose (light version) were compared. The Quantitative Descriptive Analysis evidenced that the perception of the attributes cream color, vanilla aroma, soy aroma, vanilla flavor soy flavor, sweetness and viscosity was significantly superior to the sample with sucrose $(p \leq 0,05)$. It was not found significant difference between the samples for homogeneity. The curves time-intensity parameters, Imax, Area and Ttot, for the sample sweetened with sucrose were superior in a significant form. The time to reach the maximum intensity (TImax) of sucralose sweetness in soy "milk" is higher, or either, it takes more time to be perceived.

KEY-WORDS: SOY-SENSORY ANALYSIS; SWEETENER; HIDROSSOLUBLE SOY EXTRACT.

\section{REFERÊNCIAS}

1 AOAC. Association of Official Analytical Chemistry. Official methods of analysis of AOAC International. Washington, USA, 1995. $1141 \mathrm{p}$.

2 BIRCH, G.G.; LATYMER,Z; HOLOWAY, M.O. Intensity/time relationships in sweetness: evidence for a queue hypotesis in taste chemoreception. Chem. Senses, v.5, p.63-79, 1980.

3 BONNANS, S.; NOBLE,A.C. Effect of sweetener type and sweetener and acid levels on temporal perception of sweetness, sourness and fruitness. Chem. Senses., v.18, p.272-83, 1993. 

318 de 24 de novembro de 1995. Aprova o uso de sucralose com a função de edulcorante em alimentos e bebidas dietéticas. Diário Oficial [da] República Federativa do Brasil, Brasília, n. 227, p.19406, 28 nov. 1995.

5 CÂNDIDO, L.M.B.; CAMPOS, A.M. Alimentos para fins especiais: dietéticos. São Paulo: Varela, 1996. 423 p.

6 CARDELLO, H.M.A.B.; SILVA, M.A.A.P. da; DAMÁSIO, M.H. Programa Sistema de coleta de dados tempo-intensidade (SCDTI). In: SIMPÓsIO IBEROAMERICANO DE ANÁLISE SENSORIAL, 1., 1996, Campinas. Resumos... Campinas: FEA, 1996.

7 CLIFF, M.; HEYMANN, H. Development and use of time-intensity methodology for sensory evaluation: a review. Food Res. Int., v.26, p.375-85, 1993.

DAMÁSIO, M.H.; COSTELL, E. Análisis sensorial descriptivo: generación de descriptores y selección de catadores. Rev. Agroquím. Tecnol. Alim., v.3, n. 1/2, p.165-78, 1991.

9 DUTRA DE OLIVEIRA, J.E. Uso da soja como feijão. In: MIYSAKA, S.; MEDINA, J.C. A soja no Brasil. Campinas: ITAL, 1981. p. 847-9.

10 DUTRA DE OLIVEIRA, J.E.; SCATENA, L.; OLIVEIRA NETO, N.; DUATRE, G.G. The nutritive value of soy milk in malnourish children: a comparative study. J. Pediatr., v.69, p.670-5, 1996.

11 FRANCO, G. Tabela de composição química dos alimentos. 9.ed. São Paulo: Atheneu, 1996. 307 p.

12 KETELSEN, S.M.; KEAY, C.L.; WIET, S.G. Time-intensity parameters of selected carbohydrate and high potency sweeteners. J.Food Sci., v.58, p.1418-21, 1993.

13 MEILGAARD, M.; CIVILLE, C. Measuring responses. In: SENSORY evaluation techniques. $3^{\text {rd }}$ ed. New York: CRC, 1999. 281 p.

14 MILLER, G.A. Sucralose. In: NABORS, L.B.; GELARDI, R.C. Alternatives sweeteners. $2^{\text {nd }}$ ed. New York: Marcel Dekker, 1991. p.173-195.

15 MORAIS, A.A.; SILVA, A.L. A soja: suas aplicações. Rio de Janeiro: Medsi, 1996. $259 \mathrm{p}$.

16 MOSKOWITZ, H.R. Product testing and sensory evaluation of foods. Westport: Food e Nutrition Press, 1983. 605 p.

17 MUÑOZ, A.M.; PANGBORN, R.M.; NOBLE, A.C. Sensory and mechanical attributes of gel texture. II. Gelatin, sodium alginate and kappa-carrageenan gels. J. Texture Stud., v.17, p.17-36, 1986. 
18 SAS Institute. SAS User's Guide: statistics. Cary, USA, 2001.

19 STONE, H.; SIDEL, J. Sensory evaluation practices. New York: Academic Press, 1993. $338 \mathrm{p}$.

\section{Agradecimentos}

Os autores agradecem à FAPESP e ao CNPq pelo apoio financeiro recebido. 